\title{
THE FOLIAGE DENSITY EQUATION REVISITED
}

\author{
JOHN BORIS MILLER
}

(Received 24 January 1985; revised 15 August 1985)

\begin{abstract}
The foliage density equation is the means by which the foliage density $g$ in a leaf canopy, as a function of the angle of inclination of the leaves, is to be estimated from discrete data gathered using photometric methods or point quadrats. It is an integral equation relating $f$, a function of angle estimated from measurements, to the unknown function $g$. The explicit formula for $g$ is known and depends upon $f$ and its first three derivatives; the operator $f \mapsto g$ is unbounded, and the problem is ill posed.

In this paper we give the form of $g$ when $f$ is a trigonometric polynomial, extending earlier results due to J. R. Philip. This provides a means of estimating $g$ without directly estimating the derivatives of $f$ from numerical data. To assess the reliability of the method we discuss the convergence of Fourier series representations of $f$ and $g$.
\end{abstract}

\section{Introduction}

Lately there has been renewed interest in the foliage density equation; this is a Fredholm equation of the first kind on the interval $\left[0, \frac{1}{2} \pi\right]$,

$$
f(\beta)=\int_{0}^{\pi / 2} K(\alpha, \beta) g(\alpha) d \alpha \quad\left(0 \leqslant \beta \leqslant \frac{1}{2} \pi\right)
$$

with the rather complicated kernel function $K$ given in (5) below. The equation relates two functions $f$ and $g, f$ being deemed known and $g$ unknown. The function $f$, called the contact frequency function, is estimated from numerical data obtained from a specified spatial region of the canopy of leaves of a plant (for example, using point quadrats); the function $g$, called the foliage density function, describes the foliage density distribution: $g(\alpha) d \alpha$ is the contribution to foliage density due to foliage inclined at angles between $\alpha$ and $\alpha+d \alpha$ to the horizontal. The form of $K$ is due to J. Warren Wilson and J. E. Reeve [8], the integral

\footnotetext{
'Department of Mathematics, Monash University, Clayton, Victoria 3168

(C) Copyright Australian Mathematical Society 1986, Serial-fee code 0334-2700/86
} 
equation to J. R. Philip [7]. The author gave an explicit solution to (1) in J. B. Miller [5] in 1964. However, since this expresses $g$ as an integral involving $f$ and its first three derivatives (namely $g(\alpha)=\mathfrak{E} f(\alpha)$ in (7) below), its application presents the problem of estimating $f, f^{\prime}, f^{\prime \prime}$ and $f^{\prime \prime \prime}$ from the numerical data for $f$, with all the likelihood of error latent in that process.

It was separately shown in Miller [6] that the average foliage density

$$
F=\int_{0}^{\pi / 2} g(\alpha) d \alpha
$$

could be calculated directly from $f$ without estimating the derivatives, since

$$
F=2 \int_{0}^{\pi / 2} f(\beta) \cos \beta d \beta
$$

More recently R. S. Anderssen, D. R. Jackett and D. L. B. Jupp in [1], [2], and [3] have considered more general functionals than (2) on the foliage density function, expressing them as functionals on the contact frequency function using (7), (8) below, and thereby making them accessible to computation.

In this paper we make a further contribution to the problem of estimating the form of $g$ from $f$. In brief, it is shown that if $f$ is a trigonometric polynomial of an appropriate type, then $g$ is also, and the relation between their coefficients is found. Thus if we have an estimate of $f$ from numerical data, and fit a trigonometric polynomial to these data, then we can calculate a trigonometric polynomial approximation to $g$. This extends partial results of Philip in [7]. No estimating of third derivatives from the data is required. However, the underlying problem is ill-posed, because $\mathfrak{S}$ is unbounded. The error in using the trigonometric polynomial to estimate $g$ cannot be found directly; instead, we obtain a result (Theorem 3) which gives a rate of convergence of the Fourier series of $f$ sufficient to ensure the convergence of the transformed Fourier series to $g$.

The analysis of the action of $\mathfrak{G}$ on trigonometric polynomials and series thus provides some insight into the extent of the ill-position, which has not been analysed in detail previously. An alternative response to the ill-position is that of Anderson, Jackett and Jupp, who advocate the use of functionals on $g$ rather than estimates of $g$. Their results also have their origin in the unbounded operator $\mathfrak{S}$. They also discuss in detail the practical importance of the foliage density equation and its solution.

In the course of the paper we obtain formulae for the elements of the matrix $\Lambda$ which transforms the sequence of Fourier coefficients of $f$ to that of $g$ (Theorem 1 ); these may be of practical assistance to workers in the field, if they are used with due circumspection.

Caveat. While there exist rigorous proofs for most assertions in the paper, there are two points where it has been necessary to seek the aid of a computer to 
resolve matters of algebra; the conclusions here cannot be said to be proved, but merely circumstantial. See equations (29), (30), and (47).

\section{The integral equation and operators $\mathscr{R}, \mathscr{Q}$}

The integral equation (1), to be solved for $g$, determines an integral operator, which we denote by $\Re$. Thus the foliage density equation and the operator $\Re$ are defined as follows:

$$
f(\beta)=(\Re g)(\beta)=\int_{0}^{\pi / 2} K(\alpha, \beta) g(\alpha) d \alpha \quad\left(0 \leqslant \beta \leqslant \frac{1}{2} \pi\right)
$$

where the kernel $K$ is given by

$$
\begin{gathered}
K(\alpha, \beta)= \begin{cases}\cos \alpha \sin \beta & (\text { if } \alpha \leqslant \beta), \\
\cos \alpha \sin \beta\left\{1+\frac{2}{\pi}(\tan \theta(\alpha, \beta)-\theta(\alpha, \beta))\right\} & (\text { if } \alpha \geqslant \beta),\end{cases} \\
\theta(\alpha, \beta)=\cos ^{-1}(\tan \beta / \tan \alpha) \quad(\text { for } \alpha \geqslant \beta) .
\end{gathered}
$$

The solution of this equation likewise determines an operator, which we write as $\mathfrak{S}$ :

$$
\begin{aligned}
(\mathfrak{Q} f)(\alpha)= & -\tan \alpha \sec ^{3} \alpha \int_{\alpha}^{\pi / 2}\left(\tan ^{2} \beta-\tan ^{2} \alpha\right)^{-1 / 2} \\
& \times \frac{d}{d \beta}\left\{\left(f(\beta)+f^{\prime \prime}(\beta)\right) \cos ^{3} \beta\right\} d \beta \quad\left(0 \leqslant \alpha \leqslant \frac{1}{2} \pi\right) .
\end{aligned}
$$

Thus in suitable circumstances we have

$$
f=\Re g, \quad g=\mathfrak{Q} f .
$$

More precisely, there are the following results from Miller [5] ${ }^{\dagger}$ We shall work where possible in $L^{1}\left[0, \frac{1}{2} \pi\right]$ (briefly, $L^{1}$ ); the following discussion concerns arbitrary functions, not having regard to their phytological interpretations.

$1^{\circ}$. If $g \in L^{1}$ and $f$ is defined by $f=\Omega g$, then $f$ is differentiable on $\left[0, \frac{1}{2} \pi\right]$, and

$$
\begin{array}{r}
\frac{\pi}{2} \frac{d}{d \beta}(f(\beta) / \sin \beta)=-(\sin \beta)^{-2} \int_{\beta}^{\pi / 2} g(\alpha) \cos \alpha \sqrt{\tan ^{2} \alpha-\tan ^{2} \beta} \cdot d \alpha, \\
\left(0<\beta<\frac{1}{2} \pi\right),
\end{array}
$$

and

$$
f^{\prime}(0)=f^{\prime}\left(\frac{1}{2} \pi\right)=0
$$

\footnotetext{
${ }^{\dagger}$ In that paper it was only asserted that $f^{\prime}(0+0)=f^{\prime}\left(\frac{1}{2} \pi-0\right)=0$; but the stronger assertion (10) can be verified without great difficulty.
} 
moreover $f^{\prime \prime}(\beta)$ exists for almost all $\beta$ in $\left[0, \frac{1}{2} \pi\right], f^{\prime \prime}$ is measurable, and

$$
\frac{\pi}{2} \cos ^{3} \beta\left(f(\beta)+f^{\prime \prime}(\beta)\right)=\int_{\beta}^{\pi / 2}\left(\tan ^{2} \alpha-\tan ^{2} \beta\right)^{-1 / 2} \cos \alpha g(\alpha) d \alpha .
$$

Thus $\Re$ certainly maps into $L^{1}$. Since $K$ is bounded on the square $\left[0, \frac{1}{2} \pi\right]^{2}, \Re$ is a bounded linear operator on $L^{1}$.

$2^{\circ} . \Omega$ is one-one on $L^{1}$.

$3^{\circ}$. If $f$ is any twice-differentiable function on $\left[0, \frac{1}{2} \pi\right]$ such that $f^{\prime \prime}$ is absolutely continuous and

$$
f^{\prime}(0)=f^{\prime}\left(\frac{1}{2} \pi\right)=0
$$

then $\mathfrak{B} f \in L^{1}$ and

$$
\mathfrak{R} \mathfrak{Q} f=f .
$$

Thus if $f$ has the stated properties then the function $g=\mathfrak{Q} f$ is the unique solution in $L^{1}$ of the equation $\mathbb{R} g=f$.

These results show that the contact frequency function $f$ must be assumed to be sufficiently smooth to possess a third derivative in $L^{1}$, and must moreover satisfy (10). Not every such function $f$ can arise as a contact frequency function, since there is the further condition $g(\alpha) \geqslant 0$ for $0 \leqslant \alpha \leqslant \frac{1}{2} \pi$ to be satisfied. We do not examine that condition in this paper.

While $\mathscr{R}$ is bounded, its inverse $\mathscr{G}$ is not, and this fact is the main source of difficulty in the analysis.

In view of the above results, we introduce the following function spaces:

$$
X=\left\{f \in C^{1}\left[0, \frac{1}{2} \pi\right]: f^{\prime}(0)=f^{\prime}\left(\frac{1}{2} \pi\right)=0, f^{\prime \prime}\right. \text { exists a.e. }
$$

$$
\begin{array}{r}
\text { on } \left.\left[0, \frac{1}{2} \pi\right] \text { and is measurable }\right\} \text {, } \\
Y=\left\{f \in X: f^{\prime \prime} \text { is absolutely continuous on }\left[0, \frac{1}{2} \pi\right]\right\} .
\end{array}
$$

Then

$$
Y \subset X, \quad \mathfrak{R}: L^{1} \rightarrow X, \quad \mathfrak{Q}: Y \rightarrow L^{1}
$$

and

$$
\Re \mathfrak{Q}=\widetilde{\Im}_{Y}, \text { the identity on } Y \text {. }
$$

On a suitable subspace $Z$ of $L^{1}$ we also have $\mathfrak{E} \Re=\mathfrak{\Im}_{Z}$. It can be shown that

$4^{\circ} . \mathfrak{Q} \Re g=g$ if, for example, $g$ satisfies the conditions

(i) $g^{\prime}$ exists and is continuous on $\left(0, \frac{1}{2} \pi\right)$,

(ii) $g(\alpha)=o\left(\sec ^{3} \alpha\right)$ as $\alpha \rightarrow \frac{1}{2} \pi$,

(iii) $\int_{0}^{\pi / 2}|g(\alpha)| \cos ^{2} \alpha d \alpha<\infty, \int_{0}^{\pi / 2}\left|g^{\prime}(\alpha)\right| \cos ^{3} \alpha d \alpha<\infty$. 
So far as formalities are concerned, this and the ensuing analysis are simplified by changing from variables $\alpha, \beta$ to variables $x, y$ by putting

$$
\tan ^{2} \alpha=x, \quad \tan ^{2} \beta=y
$$

and introducing functions

$$
\begin{array}{cc}
p(y)=-\frac{\pi}{2} \sin ^{2} \beta \frac{d}{d \beta}\left(\frac{f(\beta)}{\sin \beta}\right) \quad\left(0<\beta<\frac{1}{2} \pi, 0<y<\infty\right), \\
q(x)=g(\alpha) /\left(2 \tan \alpha \sec ^{3} \alpha\right) \quad\left(0<\alpha<\frac{1}{2} \pi, 0<x<\infty\right) .
\end{array}
$$

Then the equation $g=\mathfrak{Q} f$ asserts that $q$ is the $1 \frac{1}{2}$ th derivative of $(2 / \pi) p$; more precisely,

$$
q(x)=\frac{2}{\pi} \int_{x}^{\infty}(y-x)^{-1 / 2} p^{\prime \prime}(y) d y
$$

(Miller [5], §3).

\section{The action of on trigonometric polynomials}

Suppose now that $f$ is a trigonometric polynomial of the form

$$
f(\beta)=F_{0}+F_{1} \cos 2 \beta+F_{2} \cos 4 \beta+\cdots+F_{N} \cos 2 N \beta .
$$

We show that then $g=\mathfrak{Q} f$ is a trigonometric polynomial of the form

$$
g(\alpha)=G_{0} \sin \alpha+G_{1} \sin 3 \alpha+G_{2} \sin 5 \alpha+\cdots+G_{N} \sin (2 N+1) \alpha
$$

and we obtain formulae for the coefficients $G_{k}$ in terms of the coefficients $F_{j}$. The result is formulated as Theorem 1 , below.

Note that $f$ in (17) does belong to $Y$, since (10) holds. Indeed, the only trigonometric sine or cosine terms whose derivatives vanish at 0 and $\frac{1}{2} \pi$ are those of the form $\cos 2 k \beta$, so (17) is the appropriate form to adopt for $f$, and moreover is completely general.

The passage by $\mathscr{Q}$ from (17) to (18) is not direct; the peculiar form of the operator $\mathfrak{G}$ makes it better able to handle even powers of sines, than cosines of multiple angles. So as a preliminary to calculating $\mathfrak{Q}(\cos 2 k(\cdot))(\alpha)$, we first express $\cos 2 k \beta$ as a polynomial in $\sin ^{2} \beta$ and then calculate $\mathscr{Q}\left(\sin ^{2 j}\right)(\alpha)$. For the first step there is the following formula:

$$
\begin{aligned}
& \cos 2 k \beta=1+k \sum_{j=1}^{k}(-1)^{\prime} \frac{2^{2 j-1}}{j}\left(\begin{array}{c}
k+j-1 \\
2 j-1
\end{array}\right) \sin ^{2 \jmath} \beta \\
&(k=0,1,2, \ldots) .
\end{aligned}
$$


Proof. Equate real parts in de Moivre's identity for $(\cos \beta+i \sin \beta)^{2 k}$; this gives

$$
\cos 2 k \beta=\sum_{j=0}^{k}(-1)^{\jmath} \sin ^{2 j} \beta \sum_{l=0}^{J}\left(\begin{array}{l}
2 k \\
2 l
\end{array}\right)\left(\begin{array}{l}
k-l \\
k-j
\end{array}\right) .
$$

For the terms $0<j(\leqslant k)$, the inside sum

$$
\begin{aligned}
& =\text { coefficient of } t^{j} \text { in }\left(1+t^{1 / 2}\right)^{2 k}(1-t)^{-(k-\jmath+1)} \\
& =\text { coefficient of } t^{j} \text { in }\left(1+t^{1 / 2}\right)^{k+\jmath-1}\left(1-t^{1 / 2}\right)^{-(k-\jmath+1)} \\
& =\sum_{l=0}^{2 \jmath}\left(\begin{array}{c}
k+j-1 \\
l
\end{array}\right)\left(\begin{array}{c}
k+j-l \\
2 j-l
\end{array}\right) \\
& =\left(\begin{array}{c}
k+j-1 \\
k-j
\end{array}\right) \sum_{l=0}^{2 j} \frac{(2 j-1) !(k+j-l)}{l !(2 j-l) !} \\
& =\left(\begin{array}{c}
k+j-1 \\
k-j
\end{array}\right)\left[2^{2 \jmath}(k+j)-2 j 2^{2 \jmath-1}\right] /(2 \jmath) \\
& =\left(\begin{array}{c}
k+j-1 \\
k-j
\end{array}\right) 2^{2 \jmath-1} k / j
\end{aligned}
$$

This proof is due to G. A. Watterson. Next, we have

Lemma 1. For $j=0,1,2,3, \ldots$, and $0<\alpha<\frac{1}{2} \pi$,

$$
\begin{aligned}
\mathfrak{Q}\left(\sin ^{2 \jmath}\right)(\alpha) & =2 \pi^{1 / 2}(2 j-1) j ! \sin \alpha \sum_{l=0}^{J} \frac{(-1)^{l+1}(l+1) \cos ^{2 l} \alpha}{(j-l) ! \Gamma\left(l+\frac{1}{2}\right)} \\
& =-2(2 j-1) \sin \alpha \cdot{ }_{2} F_{1}\left(-j, 2 ; \frac{1}{2} ; \cos ^{2} \alpha\right) .
\end{aligned}
$$

In particular,

$$
\mathfrak{Q}(1)(\alpha)=2 \sin \alpha, \quad \mathfrak{Q}\left(\sin ^{2}\right)(\alpha)=2 \sin \alpha\left(-1+4 \cos ^{2} \alpha\right) .
$$

Proof. The direct route is probably also the shortest. Take $f(\beta)=\sin ^{2} \beta$ in (7); change to variables $x, y$ as in (13); after some calculation we obtain, when $j \geqslant 2$,

$$
\begin{aligned}
\mathfrak{Q}\left(\sin ^{2 \jmath}\right)(\alpha)=-\frac{1}{2} x^{1 / 2}(1+x)^{3 / 2} & \int_{x}^{\infty}(y-x)^{-1 / 2}\left\{A_{j} y^{J-2}(1+y)^{-\jmath-3 / 2}\right. \\
& -B_{J} y^{J-1}(1+y)^{-\jmath-3 / 2} \\
& \left.-C_{J} y^{J-1}(1+y)^{-j-5 / 2}+D_{j} y^{J}(1+y)^{-j-5 / 2}\right\} d y,
\end{aligned}
$$

where

$$
\begin{array}{ll}
A_{j}=2 j(2 j-1)(2 j-2), & B_{j}=6 j(2 j-1), \\
C_{j}=(2 j+1) 2 j(2 j-1), & D_{J}=3(2 j+1)(2 j-1) .
\end{array}
$$


For the integrals here, there is the formula (Erdélyi et al. [4], p. 201, (8))

$$
\begin{aligned}
\int_{x}^{\infty}(y-x)^{-1 / 2} y^{\sigma}(1+y)^{-\tau} d y \\
\quad=(1+x)^{\sigma-\tau+1 / 2} \frac{\Gamma\left(\frac{1}{2}\right) \Gamma\left(\tau-\sigma-\frac{1}{2}\right)}{\Gamma(\tau-\sigma)} \cdot{ }_{2} F_{1}\left(-\sigma, \tau-\sigma-\frac{1}{2} ; \tau-\sigma ; \frac{1}{1+x}\right),
\end{aligned}
$$

valid when $\tau-\sigma>\frac{1}{2}$ and $x>0$. Using this on each of the four terms and combining the results, we end up, after more heavy algebra, with the equation (20). Separate verifications show that (20) holds also when $j=0$ and 1 , giving (21).

The terms $\sin \alpha \cos ^{2 /} \alpha$ in (20) can be expressed as trigonometric polynomials of the required type by the formula

$$
\sin \alpha \cos ^{2 l} \alpha=\sum_{m=0}^{l}\left(\begin{array}{c}
2 l+1 \\
l+m+1
\end{array}\right) 2^{-2 l} \frac{2 m+1}{2 l+1} \sin (2 m+1) \alpha \quad(l=0,1,2, \ldots) .
$$

So finally we have, using (19), (20) and (23):

$$
\begin{aligned}
g(\alpha)= & (\mathfrak{E} f)(\alpha) \\
= & \mathfrak{Q}\left(\sum_{k=0}^{N} F_{k} \cos 2 k \beta\right)(\alpha) \\
= & \sum_{k=0}^{N} F_{k}\left(1+k \sum_{j=1}^{k}(-1)^{\prime} \frac{2^{2 j-1}}{j}\left(\begin{array}{c}
k+j-1 \\
2 j-1
\end{array}\right) \mathfrak{S}\left(\sin ^{2 \jmath}\right)(\alpha)\right) \\
= & \sum_{k=0}^{N} F_{k}-\sum_{k=1}^{N} k F_{k} \sum_{j=1}^{k}(-1)^{j} \frac{2^{2 j-1}}{j}\left(\begin{array}{c}
k+j-1 \\
2 j-1
\end{array}\right) \cdot 2(2 j-1) \\
& \times \pi^{1 / 2} j ! \sin \alpha \sum_{l=0}^{j} \frac{(-1)^{l+1}(l+1) \cos ^{2 l} \alpha}{(j-l) ! \Gamma\left(l+\frac{1}{2}\right)} \\
= & \sum_{k=0}^{N} F_{k}-\sum_{k=1}^{N} k F_{k} \sum_{j=1}^{k}(-1)^{j} \frac{2^{2 j-1}}{j}\left(\begin{array}{c}
k+j-1 \\
2 j-1
\end{array}\right) 2(2 j-1) \\
& \times \pi^{1 / 2} j ! \sum_{l=0}^{j} \frac{(-1)^{l+1}(l+1)}{(j-l) ! \Gamma\left(l+\frac{1}{2}\right)} \sum_{m=0}^{l}\left(\begin{array}{c}
2 l+1 \\
l+m+1
\end{array}\right) 2^{-2 l} \frac{2 m+1}{2 l+1} \sin (2 m+1) \alpha .
\end{aligned}
$$


By judicious rearrangement of the summations we can express this in the form of a sum with respect to $m$, from 0 to $N$, that is, in the form (18), and so obtain a formula for the coefficients $G_{m}$ in terms of the $F_{k}$. The result is (after a trivial change of notation)

$$
G_{j}=\sum_{k=j}^{N} \lambda_{j k} F_{k} \quad(j=0,1, \ldots, N)
$$

where

$$
\lambda_{0 k}=2 \text { for } k=0,1,2, \ldots, N
$$

and

$$
\lambda_{j k}=\frac{-4 \pi^{1 / 2}(j+1) ! k(k+j-1) !}{(2 j) ! \Gamma\left(j-\frac{1}{2}\right)(k-j) !}{ }_{3} F_{2}\left(-k+j, j, k+j ; j-\frac{1}{2}, 2 j+2 ; 1\right)
$$

for $j=1,2, \ldots, N$ and $j \leqslant k \leqslant N$. Put $\lambda_{j k}=0$ for $k<j$.

Write $r=k-j$, and $S_{r}(j)$ for the generalized hypergeometric function (evaluated at 1 ) which appears here, so that

$$
\lambda_{J, j+r}=\frac{-4 \pi^{1 / 2}(j+1) !(j+r)(2 j+r-1) !}{(2 j) ! \Gamma\left(j-\frac{1}{2}\right) r !} S_{r}(j) \quad(j \geqslant 1, r \geqslant 0) .
$$

$S_{r}(j)$ is the sum of a finite series. Although the parameters in it do not reduce the ${ }_{3} F_{2}$ to any of the standard types for which there is a known representation as a rational fraction of gamma functions, nevertheless such a representation seems to exist. Using a Burroughs B6700, B. J. Milne has obtained the following formulae, valid at least for $r=0,1, \ldots, 12$ and all $j \geqslant 1$. There are separate forms for $r=2 s+1$ and $r=2 s, s$ integral, $\geqslant 1$ :

$$
\begin{gathered}
S_{2 s+1}(j)= \\
-\frac{1^{2} \cdot 3^{2} \cdot 5^{2} \cdots(2 s-1)^{2}(2 s+1)}{2^{2 s+1}\left(j^{2}-\frac{1}{4}\right)(j+1)\left(j+\frac{3}{2}\right)^{2}\left(j+\frac{5}{2}\right)^{2} \cdots\left(j+\frac{2 s-1}{2}\right)^{2}\left(j+\frac{2 s+1}{2}\right)} \\
S_{2 s}(j)=S_{2 s+1} \cdot \frac{2 j^{2}+(4 s+1) j+4 s^{2}-1}{(2 s-1)(2 s+1)}
\end{gathered}
$$


The quadratic factor in (30) has real linear factors if and only if $s=0$ or 1 . I have not been able to prove (29) or (30) for arbitrary $s$, but it is reasonable to assume that they are true generally, and we proceed on that assumption. Substituting in (28) and simplifying, we obtain formulae for $\lambda_{j k}$, namely

$$
\begin{aligned}
\lambda_{j, j+2 s+1}= & 2^{2 j+1}(2 j+1) \frac{(2 s) !}{(s !)^{2}}(j+2 s+1) \frac{(j+s) !^{2}}{(2 j+2 s+1) !}, \\
\lambda_{J, j+2 s}= & 2^{2 j}(2 j+1) \frac{(2 s) !}{(s !)^{2}(2 s-1)} \\
& \times \frac{(j+2 s)(j+s) !^{2}\left(2 j^{2}+(4 s+1) j+4 s^{2}-1\right)}{(j+s) \cdot(2 j+2 s+1) !}
\end{aligned}
$$

for $s=0,1,2, \ldots$ To sum up, we have

THEOREM 1. Let $f$ be the trigonometric polynomial (17). Then $g$, defined to be $\mathfrak{Q} f$ as in (7), is equal to the trigonometric polynomial (18); the coefficients $G_{j}$ are given in terms of the coefficients $F_{k}$ by

$$
G_{j}=\sum_{k=j}^{N} \lambda_{j k} F_{k} \quad(j=0,1,2, \ldots, N),
$$

where the $\lambda_{j k}$ are given by (26) and (27), and more simply by (31) and (32). In particular,

$$
G_{0}=2 \sum_{k=0}^{N} F_{k}=2 f(0)
$$

Some remarks are in order.

$1^{\circ}$. The numbers $\lambda_{j k}$ do not depend upon $N$. Even if the terms of the sequence $F_{0}, F_{1}, \ldots$, do not depend upon $N$, nevertheless the numbers $G_{0}, G_{1}, \ldots$ depend upon $N$.

$2^{\circ}$. The top left-hand corner of the infinite matrix $\Lambda=\left(\lambda_{j k}\right)$ is shown in Table 1. The diagonal elements

$$
\lambda_{j j}=-\frac{2^{2 j-1} j(j+1)(j-1) !^{2}}{(2 j-2) !} \quad(j=1,2, \ldots)
$$

are negative, all elements for $j<k$ are positive. From (31) and (32) we deduce that

$$
\lim _{r \rightarrow \infty} \lambda_{j r}=2(2 j+1)
$$




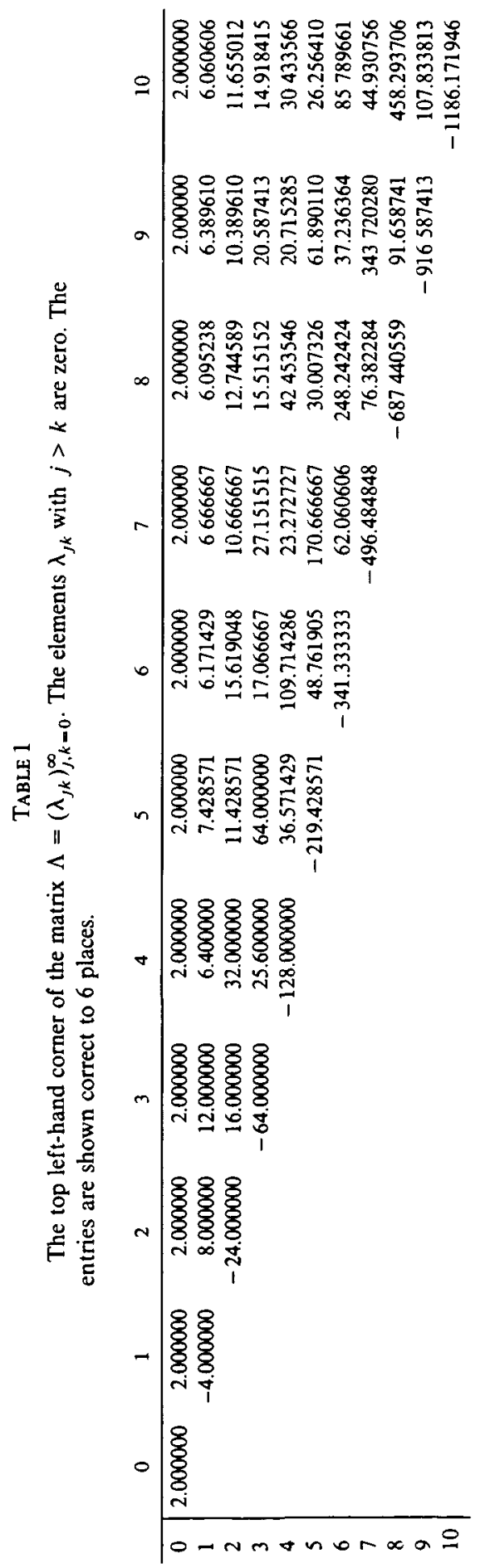


whereas from (34)

$$
\lambda_{j} \sim-2 \pi^{1 / 2} j^{3 / 2} \text { as } j \rightarrow \infty
$$

Table 2 shows the form of $\Lambda$ in the vicinity of a diagonal element: $-\mu$ denotes $\lambda_{j j}$, for a fixed $j$. All the elements shown are thus of order $j^{3 / 2}$ or $j^{1 / 2}$ as $j \rightarrow \infty$.

TABLE 2

$$
\begin{array}{cccc}
\frac{2 j-3}{2(j+1) j} \mu & \frac{2 j-3}{4(j+1)} \mu & \frac{(2 j-3)}{2(2 j-1) j} \mu & \frac{(2 j-3)(j+2)\left(2 j^{2}+j+5\right)}{8(2 j-1) j(j+1)(2 j+1)} \mu \\
-\frac{2 j-3}{2(j+1)} \mu & \frac{1}{j+1} \mu & \frac{1}{2} \mu & \frac{j+2}{(2 j+1)(j+1)} \mu \\
0 & -\mu & \frac{2}{2 j-1} \mu & \frac{j+2}{2 j-1} \mu \\
0 & 0 & -\frac{2 j+4}{2 j-1} \mu & \frac{4(j+2)}{(2 j-1)(2 j+1)} \mu
\end{array}
$$

$3^{\circ}$. If the leading terms in (25) dominate, we can write it as

$$
\begin{aligned}
G_{j}=\mu\left(-F_{j}+\frac{2}{2 j-1} F_{j+1}+\right. & \frac{j+2}{2 j-1} F_{j+2}+\frac{2(j+3)}{(2 j-1)(2 j+3)} F_{j+3} \\
& \left.+\frac{(j+4)\left(2 j^{2}+9 j+15\right)}{2(2 j-1)(2 j+3)(2 j+5)} F_{j+4}+\cdots\right)
\end{aligned}
$$

so formally,

$$
G_{j} \sim 2 \pi^{1 / 2} j^{3 / 2}\left(-F_{j}+\frac{1}{j} F_{j+1}+\frac{1}{2} F_{j+2}+\frac{1}{2 j} F_{j+3}+\frac{1}{8} F_{j+4}+\cdots\right) .
$$

$4^{\circ}$. J. R. Philip [7] established results equivalent to the equation $g=\mathfrak{Q} f$ when $f, g$ are as in (17), (18), for the cases $N=0,1,2,3$. Our results cover the case of general $N$, and express $g$ as an ordinary trigonometric polynomial (Philip was content to express $g$ as a sum of the form $\sum_{j=0}^{N} \delta_{j} \sin \alpha \cos 2 j \alpha$ for these low values of $N$ ).

\section{4. $f$ and $g$ as Fourier series}

Suppose now that $f$ is taken to be the sum of a Fourier series

$$
f(\beta)=\sum_{k=0}^{\infty} F_{k} \cos 2 k \beta \quad\left(0 \leqslant \beta \leqslant \frac{1}{2} \pi\right)
$$


rather than a trigonometric polynomial; can $g$ be represented by a series $g(\alpha)=\sum_{j=0}^{\infty} G_{j} \sin (2 j+1) \alpha$ for suitably defined coefficients $G_{J}$ (recall that in $\S 3$ the numbers $G_{j}$ depend upon a chosen $\left.N\right)$ ?

First we make an a priori definition of the coefficients $G_{\jmath}$, different from their meanings in §3; namely,

$$
G_{j}:=\sum_{k=j}^{\infty} \lambda_{j k} F_{k}
$$

Their existence is ensured by

THEOREM 2. Let $\left(F_{k}\right)$ be any sequence of reals for which $\sum_{k} F_{k}$ converges; then for each $j=0,1, \ldots$, the series (40) converges. If $\sum_{k} k^{5 / 2}\left|F_{k}\right|$ converges then $\Sigma_{j}\left|G_{j}\right|$ converges, and so

$$
\sum_{j=0}^{\infty} G_{j} \sin (2 j+1) \alpha
$$

converges uniformly to a continuous function.

Proof. Let $\phi_{n}=\sum_{k=0}^{n} F_{k}$. An Abel transformation gives

$$
\sum_{k=j}^{M} \lambda_{j k} F_{k}=-\lambda_{j j} \phi_{j-1}+\sum_{k=j}^{M-1}\left(\lambda_{j, k}-\lambda_{\jmath, k+1}\right) \phi_{k}+\lambda_{J M} \phi_{M} .
$$

Using (31), (32) we can show that $\lambda_{\jmath, k}-\lambda_{\jmath, k+1}=O\left(k^{-2}\right)$ for fixed $j$ as $k \rightarrow \infty$; it follows that (40) converges when the sequence $\left(\phi_{n}\right)$ does.

From the definition of $G_{j}$ we have

$$
\sum_{j=0}^{\infty}\left|G_{j}\right| \leqslant \sum_{k=0}^{\infty}\left|F_{k}\right| \cdot \sum_{j=0}^{k}\left|\lambda_{j k}\right| \text {. }
$$

An asymptotic formula for the sequence of numbers

$$
D_{k}:=\sum_{j=0}^{k}\left|\lambda_{j k}\right|
$$

can be guessed with the help of a computer, as follows. If $k$ is even, $k=2 t$ $(t=1,2, \ldots)$, we get after some heavy algebra from (31), (32):

$$
\begin{aligned}
D_{2 t}= & \frac{2\left(16 t^{2}-1\right)}{4 t^{2}-1} \sum_{p=0}^{t-1} \frac{\left(\frac{3}{2}\right)_{p}(t)_{p}(-t)_{p}}{\left(\frac{1}{2}\right)_{p}\left(t+\frac{3}{2}\right)_{p}\left(\frac{3}{2}-t\right)_{p}}-\lambda_{2 t, 2 t} \\
= & \frac{2\left(16 t^{2}-1\right)}{4 t^{2}-1}{ }_{4} F_{3}\left(\frac{3}{2}, 1, t,-t ; \frac{1}{2}, \frac{3}{2}+t, \frac{3}{2}-t ; 1\right) \\
& +\frac{2^{4 t+1}(2 t+1)(4 t-1)(2 t) !^{2}}{(4 t) !}
\end{aligned}
$$


For $k$ odd, $k=2 t+1(t=0,1,2, \ldots)$, we get instead

$$
\begin{aligned}
D_{2 t+1}= & \frac{2(4 t+1)(4 t+3)}{(2 t-1)(2 t+3)} \cdot{ }_{4} F_{3}\left(\frac{3}{2}, 1, t+1,-t ; \frac{1}{2}, \frac{5}{2}+t, \frac{3}{2}-t ; 1\right) \\
& +\frac{2^{4 t+3}(2 t+1)(t+1)(2 t) !^{2}}{(4 t) !} .
\end{aligned}
$$

B. J. Milne has shown using a B7800 that in each of (45) and (46) the first term is $\sim-2 t^{2}$ as $t \rightarrow \infty$; the second term is therefore dominant in each case (as Table 1 suggests), and we find

$$
\sum_{j=0}^{k}\left|\lambda_{\jmath k}\right|-4 \pi^{1 / 2} k^{5 / 2} \quad \text { as } k \rightarrow \infty .
$$

Thus if $\sum_{k} k^{5 / 2}\left|F_{k}\right|$ converges, (43) shows that $\sum_{j}\left|G_{j}\right|$ converges.

It remains to establish sufficient conditions under which the sum (41) is $(\mathfrak{Q} f)(\alpha)$.

THEOREM 3. Let $\left(F_{k}\right)_{k \in N}$ be any sequence such that the series $\sum_{k} k^{5 / 2}\left|F_{k}\right|$ converges. Let $f$ be the $L^{1}$ function defined by

$$
f(\beta)=\sum_{k=0}^{\infty} F_{k} \cos 2 k \beta \quad\left(0 \leqslant \beta \leqslant \frac{1}{2} \pi\right)
$$

and write

$$
G_{J}=\sum_{k=j}^{\infty} \lambda_{j k} F_{k} \quad(j=0,1, \ldots) .
$$

Then for the function $g$ defined by

$$
g(\alpha)=\sum_{j=0}^{\infty} G_{j} \sin (2 j+1) \alpha
$$

we have $f=\Re g$. If moreover $\sum_{k} k^{7 / 2}\left|F_{k}\right|$ converges then $g=\mathfrak{G} f$, and $g$ is the foliage density function corresponding to $f$.

To prove this we need further notation. With $G_{j}$ and $g$ defined as stated, write now $G_{N, J}$ for the sum (25) (previously denoted by $G_{j}$ ) and write

$$
\begin{aligned}
& f_{N}(\beta)=\sum_{k=0}^{N} F_{k} \cos 2 k \beta, \\
& \tilde{g}_{N}(\alpha)=\sum_{j=0}^{N} G_{j} \sin (2 j+1) \alpha, \quad g_{N}(\alpha)=\sum_{j=0}^{N} G_{N, j} \sin (2 j+1) \alpha
\end{aligned}
$$

for these partial sums. Theorem 1 says that

$$
g_{N}=\mathfrak{Q} f_{N}, \quad f_{N}=\Re g_{N} .
$$


Let $\|\cdot\|$ denote the $L^{1}$ norm. The following lemmas use the assumptions in the theorem.

Lemma 2. For $j=0,1,2, \ldots, N$ and $k=N+1, N+2, \ldots$ we have

$$
\lambda_{J, k} \leqslant \lambda_{N, N+2}=-\lambda_{N, N} \cdot \frac{N+2}{2 N-1} .
$$

The set of numbers $\lambda_{j k}$ here is the set of elements of the matrix $\Lambda$ making up a top righthand submatrix with $\lambda_{N, N+1}$ as the lower lefthand corner element. The inequality in (53) can be read for low values of $N$ from Table 1. A laborious comparison of values of $\lambda_{j k}$ for fixed $j$ or for fixed $k$ using (31) and (32) leads eventually to the result (53). We omit the details.

LEMMA 3. $\lim _{N \rightarrow \infty}\left(\tilde{g}_{N}(\alpha)-g_{N}(\alpha)\right)=0$ uniformly for $\alpha \in\left[0, \frac{1}{2} \pi\right]$.

Proof.

$$
\begin{aligned}
\left|\tilde{g}_{N}(\alpha)-g_{N}(\alpha)\right| & \leqslant \sum_{j=0}^{N}\left|G_{j}-G_{N, j}\right| \\
& \leqslant \sum_{j=0}^{N} \sum_{k=N+1}^{\infty} \lambda_{j k}\left|F_{k}\right| \\
& \leqslant-\lambda_{N, N}\left(\frac{N+2}{2 N-1}\right)(N+1) \sum_{k=N+1}^{\infty}\left|F_{k}\right|
\end{aligned}
$$

by Lemma 2. The sum here is $o\left(N^{-5 / 2}\right)$, so (36) gives the result.

Proof of the theorem, continued. We have $\left\|g_{N}-\tilde{g}_{N}\right\| \rightarrow 0$ by Lemma 3, and $\left\|\tilde{g}_{N}-g\right\| \rightarrow 0$ by Theorem 2 , so $\left\|g_{N}-g\right\| \rightarrow 0$. Since $\Re$ is a bounded operator,

$$
\|f-\Re g\|=\lim \left\|f_{N}-\mathfrak{\Omega} g\right\|=\lim \left\|\Re g_{N}-\Re g\right\| \leqslant\|\Re\| \cdot \lim \left\|g_{N}-g\right\|=0,
$$

so $f(\beta)=\mathfrak{R} g(\beta)$ almost everywhere, in fact everywhere since both functions are continuous.

Now suppose that $\sum_{k} k^{7 / 2}\left|F_{k}\right|$ converges. This ensures that $g$ is differentiable and $g^{\prime}(\alpha)=\sum_{j=0}^{\infty}(2 j+1) G_{j} \cos (2 j+1) \alpha$, the series converging uniformly on $\left[0, \frac{1}{2} \pi\right]$. This is sufficient to ensure that the conditions (i)-(iii) of $\$ 2,4^{\circ}$ are satisfied, so from $f=\Re g$ we deduce $\mathfrak{Q} f=\mathfrak{Q} \Re g=g$. 


\section{Conclusion}

The estimation of the foliage density function $g$ from the experimentally estimated contact frequency function $f$ can be done if $f$ can be approximated to sufficient accuracy by a trigonometric polynomial (17). The calculated form of $g$, namely (18) where the $G_{j}$ 's are given by (25), should be reliable provided the sequence of coefficients $\left(F_{k}\right)$ is rapidly decreasing, say

$$
F_{k}=O\left(k^{-(9 / 2+\varepsilon)}\right)
$$

for some positive $\varepsilon$. However, because $\mathfrak{Q}$ is an unbounded operator there exists no simple inequality between the $L^{1}$ norms of $g-g_{N}$ (or $g-\tilde{g}_{N}$ ) and $f-f_{N}$ enabling the rate of convergence of the former to be deduced from that of the latter.

\section{Acknowledgements}

I must thank Dr. G. A. Watterson for the ingenious proof of the combinatorial identity leading to (19); Mr. B. J. Milne for doing the computing which leads to the formulae (29), (30) and (47); and Dr. H. Exton for advice about generalized hypergeometric functions.

\section{References}

[1] R. S. Anderssen and D. R. Jackett, "Computing the foliage angle distribution from contact frequency data", in Computational Techniques and Apphcations: CTAC 1983 (eds. J. Noye and C. Fletcher), (North-Holland, Amsterdam, 1984), 863-872.

[2] R. S. Anderssen and D. R. Jackett, "Linear functionals of foliage angle density", J. Austral. Math. Soc. Ser. B 25 (1984), 431-442.

[3] R. S. Anderssen, D. R. Jackett and D. L. B. Jupp, "Linear functionals of the foliage angle distribution as tools to study the structure of plant canopies", Aust. J. Botany 32 (1984), 147-156.

[4] A. Erdélyi et al., Higher transcendental functions, Vol. 2 (Bateman Manuscript Project, McGraw-Hill, 1953).

[5] J. B. Miller, “An integral equation from phytology", J. Austral. Math. Soc. 4 (1964), 397-402.

[6] J. B. Miller, “A formula for average foliage density", A ust. J. Botany 15 (1967), 141-144.

[7] J. R. Philip, "The distribution of foliage density with foliage angle estimated from inclined point quadrat observations", Aust. J. Bot. 13 (1965), 357-366.

[8] J. Warren Wilson (with Appendix by J. E. Reeve), "Inclined point quadrats", The New Phytologist 59 (1960), 1-8. 